\title{
Effect of the degree of protonation of amino acid dopants in Chirality amplification in DSCG Lyotropic Nematic phases
}

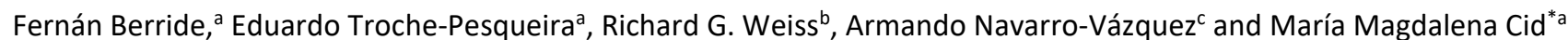

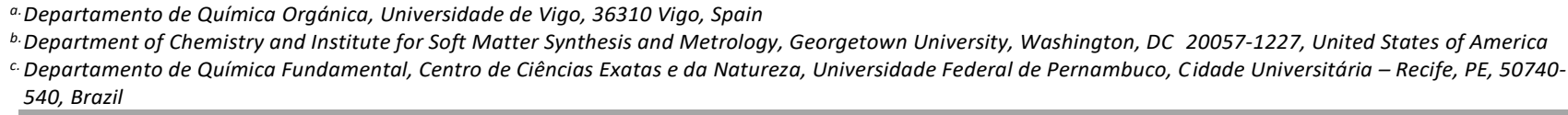

b. Department of Chemistry and Institute for Soft Matter Synthesis and Metrology, Georgetown University, Washington, DC 20057-1227, United States of America c. Departamento de Química Fundamental, Centro de Ciências Exatas e da Natureza, Universidade Federal de Pernambuco, Cidade Universitária - Recife, PE, 50740540, Brazil

Disodium Cromoglycate (DSCG), a lyotropic liquid crystal in water, is an amplifier of chirality when doped with small chiral molecules. The DSCG nematic phase in the presence of three aminoacids with different degrees of protonation: L-Alanine, L-Arginine. $\mathrm{HCl}$ and L-Arginine, presents a helicity whose sign depends on the sign of the Helical Twisting Power of the dopant.

\section{Introduction}

Disodium Cromoglycate $(\mathrm{DSCG})^{1}$ (Figure 1a) is a Lyotropic Chromonic Liquid Crystal (LCLC) in water. ${ }^{2}$ The phase diagram of the DSCG/water system shows two ordered phases, a nematic ${ }^{3}$ and a columnar hexagonal, ${ }^{4}$ which are formed by the selfassembly of DSCG into stacks. Although the details about how individual molecules aggregate to form the stacks are still

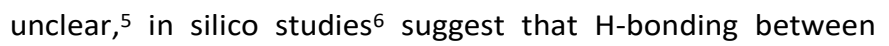
water and DSCG molecules is the main contributor, and that it is strengthened by additional interactions between aromatic rings of vicinal DSCG molecules. The formation of these stacks implies a favoured orientation and therefore induces anisotropy into the phase.
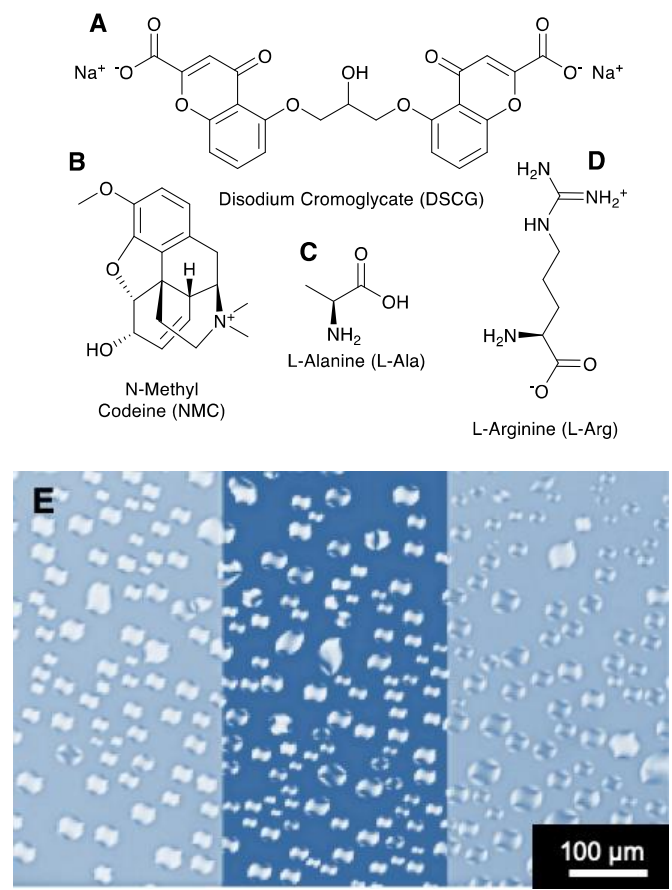

Figure 1. Molecules used in this study: A-D. E: POM microscopy of a 9wt\% DSCG with $0.8 \mathrm{wt} \% \mathrm{~N}$-Methyl Codeine at $65^{\circ}, 90^{\circ}$ and $135^{\circ}$ between polarizers.

The DSCG-LCLC system has been utilized in many applications. ${ }^{7,8,9}$ Notable among these are as an NMR aligning medium for the study of cyclodextrin host-guest complexes through residual quadrupolar splittings ${ }^{10}$ and for structural studies of small molecules through the measurement of residual dipolar couplings (RDCs). ${ }^{11,12,13,14}$

In addition, the nematic phase of DSCG has proven to be sensitive to the presence of small molecules (dopants). The effects vary from modifications in the phase transition temperature to forming chiral nematic phases if ionic salts ${ }^{15,16}$ or chiral ${ }^{17}$ dopants are added. As Labes and co-workers reported in 1984, the cholesteric chiral phase may be more or less tightly packed, depending on the helical twisting power of the chiral dopant. ${ }^{18}$

As reported for thermotropic LCs, ${ }^{19}$ DSCG solutions have shown chiral amplification through the transformation of their nematic achiral phase into a cholesteric chiral one through doping with chiral ligand-capped gold nanoparticles. ${ }^{20}$ This chiral amplification can be observed through polarized optical microscopy (POM) using wedge cells in which Grandjean steps are seen, ${ }^{21}$ or using aligned cells in which, if aligned correctly, the helical pitch of the cholesteric phase can be measured, as long as it is smaller than the cell size. ${ }^{22,23,24,25}$ Cholesteric and nematic phases are undistinguishable by POM if the pitch is too large to fully encompass the cell.

The formation of tactoids, which are prolate confined geometries, forces a twist of the director of a nematic phase in both directions, leading to a racemic tactoid mixture. ${ }^{26}$ Still, chirality can be assessed through the observation of tactoids. ${ }^{27,28}$ In 2017, we reported the observation of only one of the enantiomeric tactoid pair in a sample of DSCG doped with a small amount of $N$-methyl codeine (Figure $1 \mathrm{~b}$ and $1 \mathrm{e}$ )..$^{29}$ Very recently, chirality has also been induced in DSCG confined in spherical geometries within a matrix (as shown by the emergence of Frank-Pryce textures). ${ }^{30}$

With the aim to further broaden the scope of the use of DSCG as a chiral amplification agent, we decided to study in detail the relationship between the structure of the dopant, particularly its molecular charge and protonation state, and the degree of chiral amplification observed. The chosen systems were selected on the bases of hydrophilicity, availability in their enantiomerically pure forms and of low molecular weight. Based on those criteria, L-Alanine, L-Arginine and L-Arg. $\mathrm{HCl}$ (Figure $1 \mathrm{c}$ and $1 \mathrm{~d}$ ) were selected as a structurally related set. The DSCG chiral amplification for these amino acids was studied at several concentrations. L-Alanine is a good initial model 
because the couple DSCG_L-Ala had already been studied. ${ }^{18,28}$ L-Arginine is the amino acid that renders the highest helical twisting power ${ }^{18}$ with opposite sign of that of L-Alanine. Hence, comparing differences between these dopants can assist our understanding of the system.

The chirality enhancement was studied by means of Polarized Optical Microscopy (POM) and Circular Dichroism (CD) Spectroscopy, ${ }^{31,32}$ while the stability of the aforementioned chiral phases was assessed by ${ }^{2} \mathrm{H}$ NMR experiments. ${ }^{33}$

\section{Materials and Methods}

Cromolyn disodium salt hydrate $\left(\mathrm{C}_{23} \mathrm{H}_{14} \mathrm{Na}_{2} \mathrm{O}_{11} \cdot \mathrm{H}_{2} \mathrm{O}\right.$; $\mathrm{MW}=512.33 \mathrm{Da}$ as anhydrous) was purchased from Cofares (purity $>99.0 \%$ ); L-alanine; L-Arginine, L-Arginine $\cdot \mathrm{HCl}$ and $\mathrm{NaCl}$, were purchased from Sigma Aldrich. Samples were prepared by weight, first weighing the solid compounds, the $\mathrm{NaCl}$ with its molarity and adding the solvent at the end to a total of $1 \mathrm{~g}$ of sample. Thus, as example, the following samples were examined: DSCG 14wt\% + L-Ala 12wt\% (DSCG14_Ala12), DSCG 14wt\% + L-Ala 5wt\% (DSCG14_Ala5), DSCG 7wt\% + L-Ala 12wt\% $+\mathrm{NaCl}$ 0.3M (DSCG7_Ala12), and DSCG 7wt\% + L-Ala 5wt\% + $\mathrm{NaCl}$ 0.3M (DSCG7_Ala5).

\section{Polarized optical microscopy methodologies}

Samples were dropcast between a slide and a cover slide, which were then sealed using nail polish. Observation were made under polarized light with a NIKON E-800 optical microscope with crossed polarizers and connected to a Linkam TMS98 hot stage and a Linkam THMS central processor, using a 10x augmentation lens.

The POM textures were obtained by drop-casting the sample between two cover slides, heating them until they were full isotropic, and allowing them to cool slowly until they reached room temperature, so that the different patterns could be examined.

In the case of aligned cells, samples were placed in the side of a Standish LCD cell with a $10 \mu \mathrm{m}$ gap at $40{ }^{\circ} \mathrm{C}$, allowing the isotropic sample to flow inside the cell through capillary forces. Measurements in the images were done through ImageJ 1.52a. ${ }^{34}$

\section{NMR methodologies}

${ }^{1} \mathrm{H}$ and ${ }^{2} \mathrm{H}$ DSCG studies were performed on a Bruker AVANCE III spectrometer at $400.16 \mathrm{MHz}$ for ${ }^{1} \mathrm{H}, 101 \mathrm{MHz}$ for ${ }^{13} \mathrm{C}$, and 60.38 $\mathrm{MHz}$ for ${ }^{2} \mathrm{H}$.

\section{Circular Dichroism methodologies}

Samples were inserted in a $2 \mathrm{~mm}$ quartz cell or drop-cast in between two sandwiched quartz plates with a $0.1 \mathrm{~mm}$ optical path and placed in a Jasco J-815 CD Spectrometer to record the CD and LD spectra. Circular dichroism and UV-vis spectra were recorded between 200-900 nm using low scanning sensitivity, data pitch of $50 \mathrm{~nm}$ and a scanning velocity of $500 \mathrm{~nm} / \mathrm{min}$. For average $C D$ measurement, $C D$ spectra were measured at different rotation angles $(\theta)$ of the sample around the optical axis. $^{35}$

\section{Results}

\section{L-Alanine}

Circular dichroism spectra of various DSCG-L-Alanine samples, DSCG14_Ala12, DSCG14_Ala5, DSCG7_Ala12, and DSCG7_Ala5, were collected (Figure 2 Top). The average CD signals for all samples were positive, whereas inversion of the Lineal Dichroism (LD) signals was observed upon $90^{\circ}$ sample rotation (which is consistent with Liquid Crystal Induced Circular Dichroism $\left.(L C I C D)^{36}\right)$. Small shifts in the wavelength of the selective reflection band and large intensity differences were observed. While the latter effect was attributed to the lack of control over the sample thickness, the shift in the wavelength of the chiral reflection band can be ascribed to different doping degrees.

Schlieren textures ${ }^{37}$ were observed for DSCG14 and DSCG7 nematic phases (Figure $2 \mathrm{a}$ and Figure $2 \mathrm{~d}$, respectively). However, when the samples were doped with L-Ala, cholesteric liquid crystals textures were found in the case of DSCG14_Ala5 (focal conical texture) and DSCG14_Ala12 (fingerprint textures), as seen in Figure $2 \mathrm{~b}$ and figure $2 \mathrm{c}$, respectively. Meanwhile, the texture of the DSCG7_Ala5 sample seems to be nematic (Figure 2e), and DSCG12_Ala5 shows a texture highly reminiscent of a Twist Grain Boundary A* phase (Figure $2 \mathrm{f}$ ). It should be noted that the liquid crystal textures of a long pitch cholesteric phase can be mistaken for a nematic achiral phase, as occurred previously to Collings et al. ${ }^{38}$
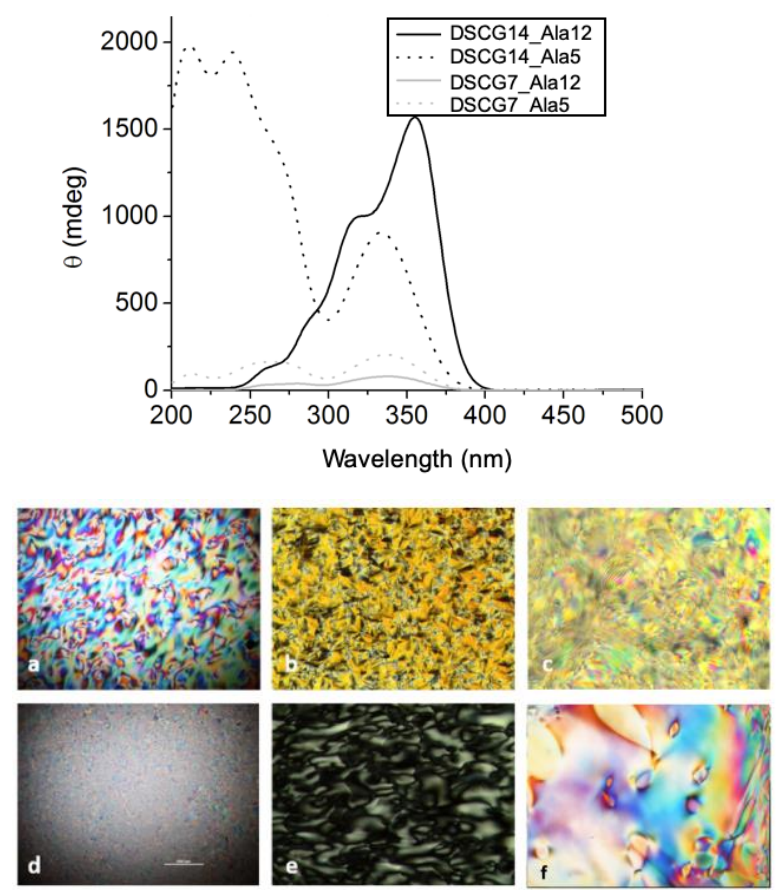

Figure 2. Top: Comparison of the CD spectra for the different DSCG_Ala samples. Bottom: Optical micrographs (10x) of different DSCG phases: a) DSCG14; b) DSCG14_Ala5; c) DSCG14_Ala12; d) DSCG7; e) DSCG7_Ala5 and f) DSCG12_Ala5

In the absence of external alignment, fingerprint textures can be observed only in regions where the helical aggregates are formed parallel to the glass surface. The pitch of the cholesteric helix, $9072 \pm 448 \mathrm{~nm}$, was calculated by measuring the repeat distances between lines in the fingerprint texture of a DSCG9_Ala 12 sample at $21^{\circ} \mathrm{C}$ (Figure 3 Top). We compared the 
inverse pitch for this sample with the expected value taking into account the reported helical twist power (HTP) of LAlanine. ${ }^{18,23,24,28}$ The inverse of the pitch measured for DSCG9_Ala 12 is larger than the values reported in the literature as a result of HTP being inversely dependent on temperature (Table 1). ${ }^{25}$ Although the average pitch of DSCG9_Ala 12 is $17 \%$ smaller than the values reported, the concentration of DSCG is $60 \%$ less, which renders the combination of DSCG with $\mathrm{NaCl}$ a good method to reduce mesogen concentration while maintaining the cholesteric phase.

The sample of DSCG9_Ala0.1 showed an homochiral set of oriented tactoids under POM when polyimide aligned cells were used (Figure 3 bottom). The twist angles between the polarizer and analyser, $\pm 10^{\circ}$ (difference with respect to crossed polarisers) to observe total light extinction, are in good agreement with those reported by. ${ }^{18}$ The Optical Activity for these tactoids, calculated as $\eta=|\tau| / c \cdot d$, where $\tau$ is the twist angle $\left(10^{\circ}\right), \mathrm{c}$ is the dopant concentration $(0.1 \mathrm{wt} \%)$, and $\mathrm{d}$ is the diameter of the tactoids $(20 \mu \mathrm{m})$, gives $\eta=5^{\circ} \times 10^{6} /(\mathrm{m} \times \mathrm{wt} \%)$. This value is $30 \%$ lower than that reported by Lavrentovich ${ }^{28}$ $\left(7.2^{\circ} \times 10^{6} /(\mathrm{m} \times \mathrm{wt})\right)$ but the sample contained $35 \%$ lower DSCG concentration; see above.

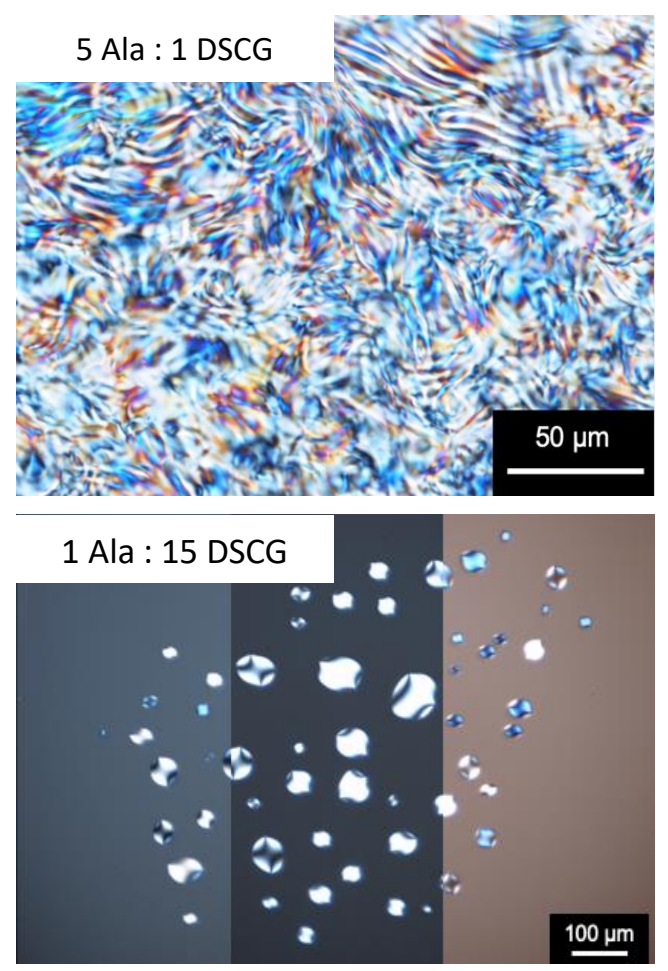

Figure 3. Top: POM image of a sample of DSCG9_Ala12 at $21^{\circ} \mathrm{C}$ showing fingerprint textures. Bottom: POM image of DSCG9_Ala0.1 at angles $80^{\circ}, 90^{\circ}$ and $100^{\circ}$ between the polarizer and analyzer.

Comparing the tactoids and cholesteric texture methods to determine the chirality of the liquid crystal, the observation of tactoids is more effective because it requires less dopant: in the fingerprints texture there are $\mathbf{5}$ molecules of L-Alanine for $\mathbf{1}$ of DSCG; while in the tactoids there is $\mathbf{1}$ molecule of L-Alanine for 15 molecules of DSCG. This result follows from the 'Sergeants and Soldiers' Principle ${ }^{39}$ and the power of tactoids as chiral amplifiers.
Table 1: Comparison of the inverse of the pitch for the DSCG9_Ala12 sample with several calculated values for DSCG15_Ala12 samples obtained from HTP fits.

\begin{tabular}{|c|c|c|c|c|}
\hline $\begin{array}{c}\text { Inverse pitch for } \\
\text { DSCG9_Ala12 } \\
\text { (Pitch =9.07 } \mu \mathrm{m})\end{array}$ & $\begin{array}{c}\mathbf{0 . 1 1 0} \\
\mathbf{~ m}^{-1}\end{array}$ & \multicolumn{4}{|l|}{} \\
\hline $\begin{array}{c}\text { Theoretical } \\
\text { inverse pitch } \\
\text { from HTP for } \\
\text { DSCG15_Ala12 }\end{array}$ & $\begin{array}{c}\text { Lee et } \\
\text { al. }^{18}\end{array}$ & $\begin{array}{c}\text { Shirai et } \\
\text { al. }^{23}\end{array}$ & $\begin{array}{c}\text { Ogolla et } \\
\text { al. }^{24}\end{array}$ & $\begin{array}{c}\text { Peng et } \\
\text { al. }^{28}\end{array}$ \\
\cline { 3 - 5 } & $0.09 \mu \mathrm{m}^{-1}$ & $\begin{array}{c}0.094 \\
\mu \mathrm{m}^{-1}\end{array}$ & $\begin{array}{c}0.13 \\
\mu \mathrm{m}^{-1}\end{array}$ & $\begin{array}{c}0.096 \\
\mu \mathrm{m}^{-1}\end{array}$ \\
\hline $\begin{array}{c}\text { Percentage } \\
\text { Difference }\end{array}$ & $\mathbf{2 2 . 5 \%}$ & $\mathbf{1 7 . 8 0 \%}$ & $\mathbf{1 4 . 9 \%}$ & $\mathbf{1 4 . 8 \%}$ \\
\hline
\end{tabular}

Powder X-ray diffraction (PXRD) experiments for DSCG14 and DSCG14_Ala12 showed that the presence of dopant leads to sharpening of the diffraction peak at $3.4 \AA$. This observation suggests that the dopant resides outside the chromonic aggregates, causing narrowing of the distribution of aggregate lengths (see figure $\mathrm{S} 1$ ), as Shirai et $\mathrm{a}^{23}$ have proposed for DSCG LCs doped with chiral dopants.

\section{L-Arginine}

Next, we studied the effect of L-Arginine and its hydrochloride as chiral dopants because i) L-Arginine has the highest HTP of the natural mono amino acids and induces the opposite handedness of that of L-Alanine and ii) L-Arginine and L-Arg. $\mathrm{HCl}$ would inform whether the protonation degree has any effect on the Cotton effects in $C D$ and for the handedness of the observed tactoids.

Indeed, CD experiments revealed that DSCG presents an induced chiral LC phase with as little as $1 \mathrm{wt} \%$ L-Arginine (DSCG9_Arg1 in Figure 4, black line) and a Cotton effect of opposite sign to the one observed with L-Alanine.

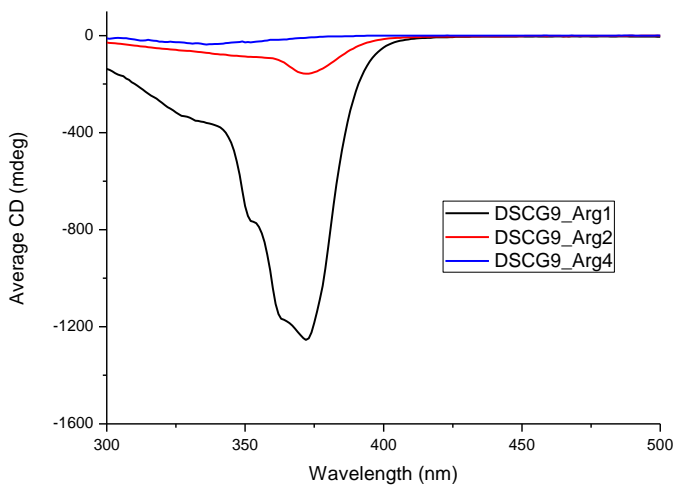

Figure 4. Average CD absorption spectra from 4 positions of DSCG9_Arg1 (blue), DSCG9_Arg2 (green) and DSCG9_Arg4 (red) at $24^{\circ} \mathrm{C}$.

However, the concentration window in which DSCG forms a cholesteric LC in the presence of L-Arginine is very narrow: at 2 wt\% (DSCG9_Arg2), the chirality of the LC phase is considerably lower than at 1 wt\% (Figure 4, red line), and at 4 wt\% (DSCG9_Arg4) the LC phase has vanished completely (Figure 4, blue line). The loss of the LC phase was confirmed by UV-Vis spectra, for which the absorbance band in the $370 \mathrm{~nm}$ region decreased with increasing concentrations of L-Arginine (see figure S2). 
In order to assess the chirality amplification, a POM study of DSCG9_Arg0.1 was carried out using aligned cells. This sample showed more aligned tactoids than its L-Alanine counterpart (Figure 5) and an opposite sign in terms of light extinction at complementary angles. According to optical measurements, the formed tactoids have an average size of $28 \mu \mathrm{m}$ and a $18^{\circ}$ twist angle, rendering an estimated optical activity of $6.4^{\circ} \times 10^{6} /(\mathrm{m} \times$ wt\%).

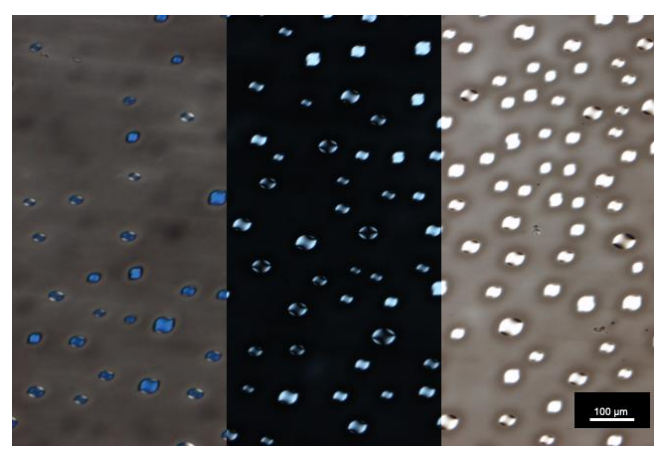

Figure 5: POM Image of the sample DSCG9_Arg0.1 at $32^{\circ} \mathrm{C}$ with polarizers crossed $72^{\circ}$, $90^{\circ}$ and $108^{\circ}$, respectively.

We confirmed the cholesteric behaviour of the DSCG doped with L-Arg when we examined the POM of DSCG9_Arg0.4 and observed the formation of a cholesteric planar phase (Figure 6 top). Interestingly, when $\mathrm{D}_{2} \mathrm{O}$ was employed instead of $\mathrm{H}_{2} \mathrm{O}$, highly aligned tactoids were observed for the same concentration (Figure 6 bottom).
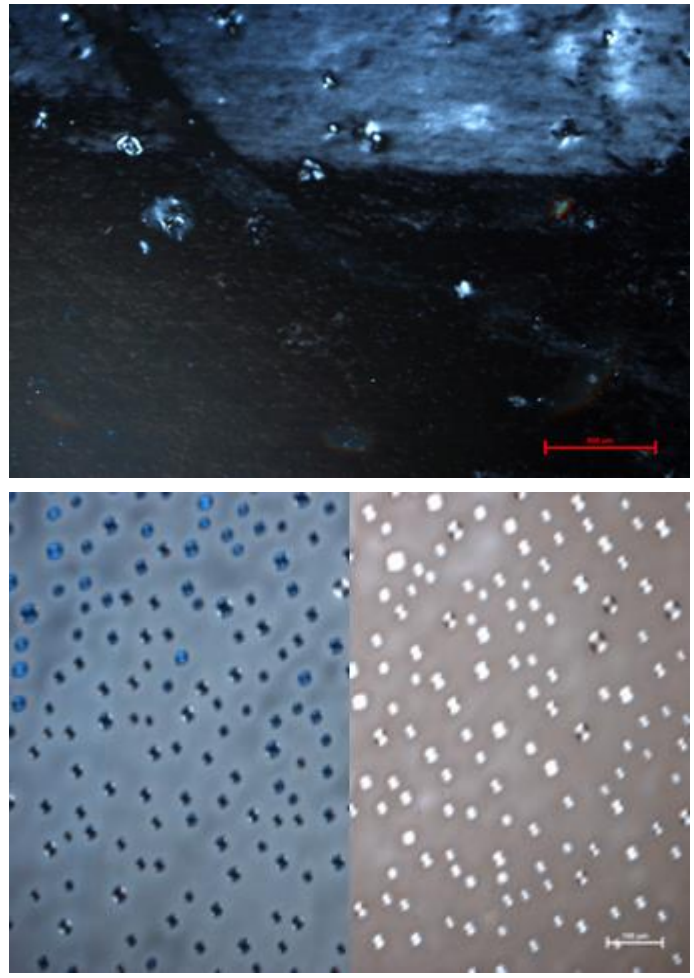

Figure 6: Top: POM Image of DSCG9_Arg0.4 $23{ }^{\circ} \mathrm{C}$. Bottom: POM Images for $9 \%$ DSCG9_Arg0.4 in $\mathrm{D}_{2} \mathrm{O}$ at $31^{\circ} \mathrm{C}$. Images with polarizers crossed $72^{\circ}$ and $108^{\circ}$, respectively

This behaviour was unexpected, but we attributed it to the weaker intermolecular bonding with water by the use of a deuterated solvent, and therefore the ability of DSCG to form helixes leading to cholesteric textures will be diminished.

\section{L-Arginine $\cdot \mathrm{HCl}$}

To evaluate the effect of the degree of protonation of the dopant, the dopant L-Arg. $\mathrm{HCl}$ was employed that would allow to explore the effect of the presence of both guanidinium and ammonium groups. Several samples containing 14 wt\% DSCG and different concentrations of L-Arg. $\mathrm{HCl}$, ranging from 1 to 4 wt \%, were prepared. Samples were doped also with $0.3 \mathrm{M} \mathrm{NaCl}$ at DSCG-7 concentrations of 1 and $2 \mathrm{wt} \% \mathrm{~L}-\mathrm{Arg} \cdot \mathrm{HCl}$, and at DSCG-9 with L-Arg. $\mathrm{HCl}$ between 0 and $4 \mathrm{wt} \%$.

POM images (Figure $7 \mathrm{a}-\mathrm{b}$ ) of DSCG14 and DSCG 14_ArgHCl4 indicated the presence of liquid crystal phases. Chiral textures were more notable in fig $7 \mathrm{~b}$, which shows cholesteric droplets characteristic of a long pitch cholesteric phase. DSCG 7 (Figure 7c-d) and DSCG9 (Figure 7e-f) samples, both undoped and L$\mathrm{Arg} \cdot \mathrm{HCl}$ doped, showed liquid crystalline textures, although no unequivocal chiral cholesteric phases.
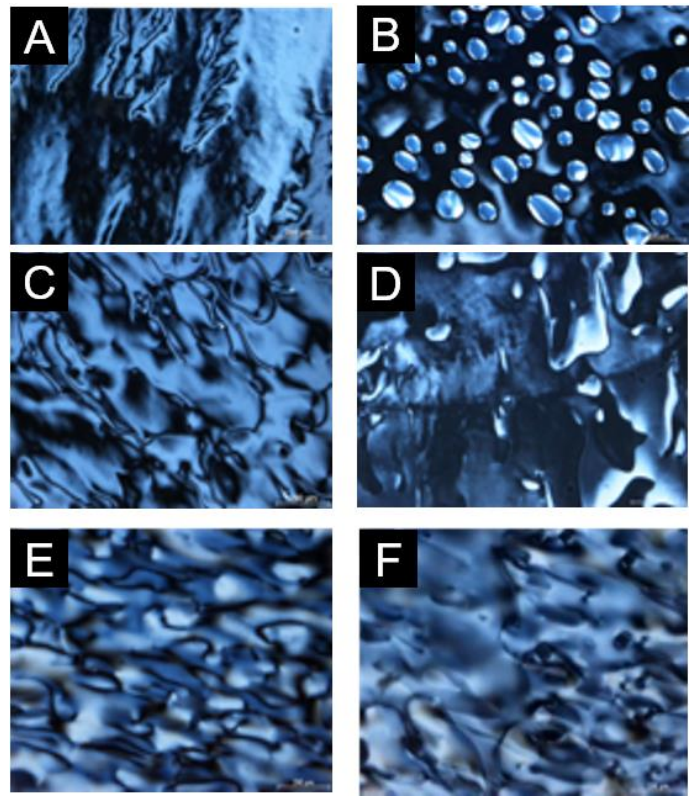

Figure 7. POM micrographs of: a) DSCG14; b) DSCG14_ArgHCl4; c) DSCG7; d) DSCG7_ArgHCl2; e) DSCG9; f) DSCG7_ArgHCl4

In an attempt to enhance the chiral sensing of this system, the $\mathrm{L}-\mathrm{Arg} \cdot \mathrm{HCl}$ concentration was reduced to $0.1 \mathrm{wt} \%$. POM using aligned cells indicated the presence of homochiral oriented tactoids (Figure 8 ) with a mean size of $27 \mu \mathrm{m}$, a $18^{\circ}$ twist angle, and an estimated optical activity $\eta$ of $6.6^{\circ} \times 10^{6} /(\mathrm{m} \times$ wt\%) (i.e., surpassing the $\eta$ estimated for L-Alanine).

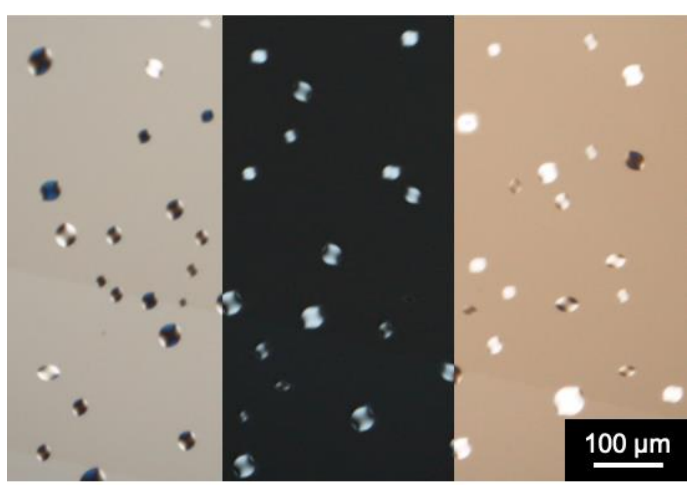

Figure 8. POM micrographs of a sample containing DSCG9_ArgHCl0.1 at $29.5^{\circ} \mathrm{C}$ with polarizers crossed $72^{\circ}, 90^{\circ}$ and $108^{\circ}$ respectively. 
Note also that the tactoid orientation under uncrossed polarizers is opposite to that of L-Alanine, which is in good agreement with the data obtained by Labes and co-workers who determined that these amino acids have reverse helical twisting powers. As we required only $0.1 \mathrm{wt} \%$ of dopant to see oriented tactoids, we needed 10 times less dopant (1 wt\%) to attain the same results as in CD spectroscopy.

The formation of a chiral phase was confirmed by Circular Dichroism spectra that show a negative Cotton effect whose sign does not change upon rotating the cell in the plane perpendicular to the incident light (Figure 9, green line).

\section{Correlation of properties}

Both the sign of the CD signal and the tactoid orientations for LArginine and L-Arginine. $\mathrm{HCl}$ are the same. Thus, the charge of the dopant does not change the chirality of the system. The average CD signal for each of the DSCG9 samples showed that L-Arg and L-Arg. $\mathrm{HCl}$ share the same CD sign, being opposite to the one observed using L-Alanine and NMC as dopants (Figure 9). This confirms previous experiments that ascribe different signs to the Helical Twisting Power of Alanine and Arginine. Based on the fact that both Ala, $\mathrm{ArgHCl}$ and $\mathrm{NMC}$ contain ammonium groups and $\mathrm{ArgHCl}$ and $\mathrm{Arg}$ contain guanidinium groups, we conclude that ammonium groups induce Positive Cotton Effects that are counterbalanced by the presence of guanidinium groups that induce, instead, Negative Cotton Effects.

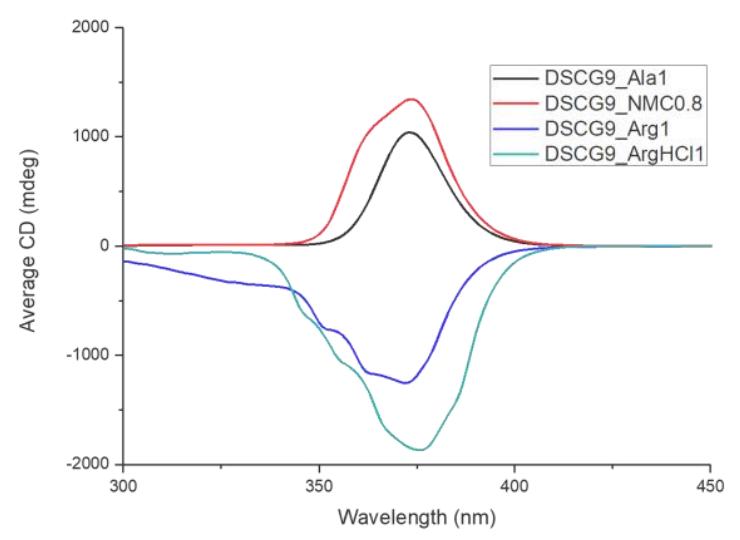

Figure 9. Average CD Spectra of DSCG9 samples doped with NMC, L-Alanine, LArginine $\cdot \mathrm{HCl}$, or L-Arginine.

To understand better the ability of each dopant as a mesophase disruptor, Deuterium NMR experiments were carried out for various doped DSCG samples (Figure 10 Top and Figures S3 and S4). DSCG7 is sensitive to dopant concentration, and only up to $4 \mathrm{wt} \%$ of L-Ala can be added before the ordered phase disappears.

DSCG14 permits up to $6 \mathrm{wt} \%$ of the dopant to be added without loss of the chiral phase that is strongly aligned as indicated by a deuterium splitting of $190 \mathrm{~Hz}$. DSCG9 showed a splitting around $120 \mathrm{~Hz}$ without loss of mesophase texture in the presence of up to $6 \mathrm{wt} \%$ of L-Alanine and $4 \mathrm{wt} \%$ of L-Arginine or L-Arginine. $\mathrm{HCl}$. ${ }^{2} \mathrm{H}-\mathrm{NMR}$ studies were performed for DSCG9 samples with 0.4 wt\% of the three aminoacids at different temperatures (Figure 10 Bottom). The results shown that ${ }^{2} \mathrm{H}$ quadrupolar splitting decreases when temperature increases, and L-Arg disturbs the nematic phase more than $\mathrm{L}-\mathrm{Arg} \cdot \mathrm{HCl}$, and the latter is more disruptive than L-Ala up to $303 \mathrm{~K}$.
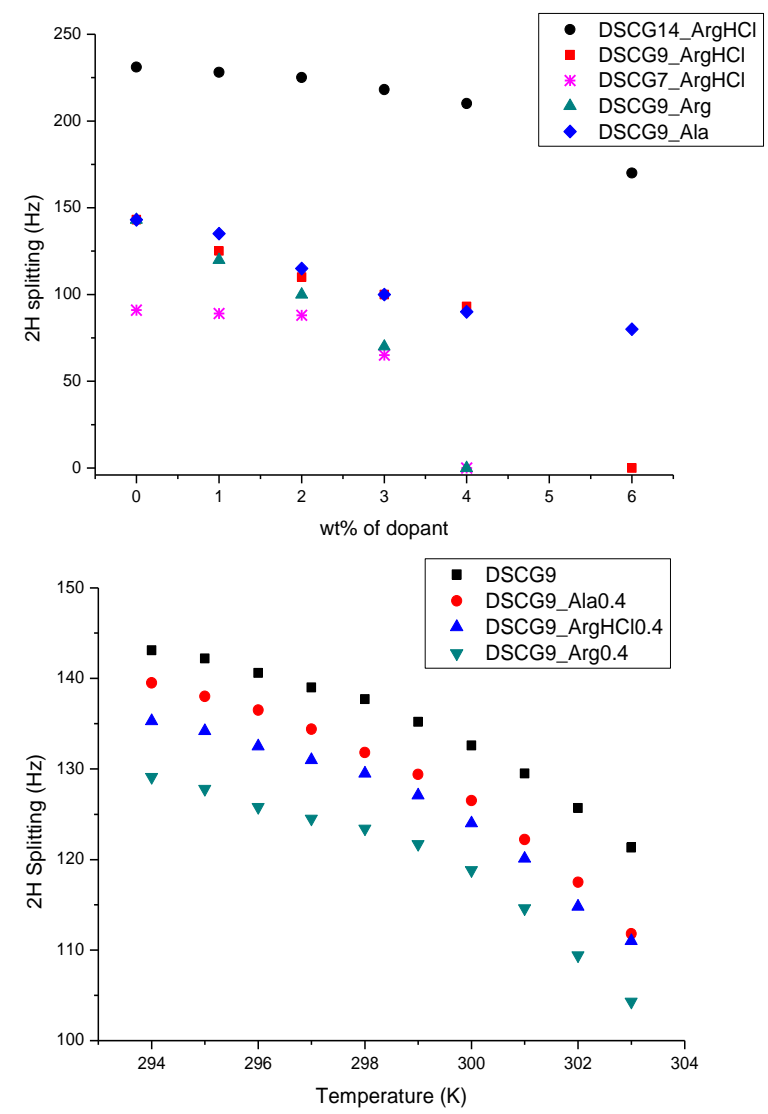

Figure 10. Top: ${ }^{2} \mathrm{H}-\mathrm{NMR}$ splitting of various DSCG + $0.3 \mathrm{M} \mathrm{NaCl}$ samples doped with different concentrations of amino acids at $298 \mathrm{~K}$. Bottom: ${ }^{2} \mathrm{H}-\mathrm{NMR}$ splitting of DSCG9 samples doped with $0.4 \mathrm{wt} \%$ of different amino acids at temperatures between 294 and 303K.

\section{Conclusions}

DSCG forms a nematic achiral phase that, upon addition of minute amounts of L-Alanine and L-Arginine as chiral dopants, transforms into a chiral cholesteric phase as shown by the textures of Polarized Optical Micrographs and CD spectra. While pitch measurements on the fingerprint texture of the Ala doped 
samples could be studied, the pitches of the L-Arg and L-Arg. $\mathrm{HCl}$ doped samples could not be measured by conventional methods. To circumvent this problem, chiral amplification was followed by observation of the tactoids formed using uncrossed polarizers. The tactoids have opposite handedness for L-Ala and $\mathrm{L}-\mathrm{Arg}$ or L-Arg. $\mathrm{HCl}$. The tactoid handedness for each of the DSCG samples doped with an amino acid has been confirmed further by induced CD signals.

Deuterium NMR experiments confirmed that the DSCG nematic phase becomes increasingly disturbed as either temperature or dopant concentration is increased. Thus, when using the nematic phase of DSCG as a chiral amplifier with chiral and hydrophilic molecules, two important factors should be kept in mind: 1) dopants should not disturb to a large extent the ordered phase; 2) an optimal temperature range from 10 to 40 ${ }^{\circ} \mathrm{C}$ should be employed.

\section{Acknowledgements}

Financial support from Marie Curie Action "International Network on Integrated Techniques in Structural Elucidation" (INTechSE), Ministerio de Economia y Competitividad of Spain (CTQ2017-85919-R) and Xunta de Galicia (ED431C 2017/70) is gratefully acknowledged. FB thanks CITACA Strategic Partnership (ED431E 2018/07) for a stipend. The authors thank RIAIDT-USC for the use of their analytical facilities.

\section{Notes and references}

1 M. J. Tasche, J. H. Uijen, R. M. Bernsen, J. C. de Jongste and J. C. van der Wouden, Thorax, 2000, 55, 913-20.

2 J. Lydon, in Handbook of Liquid Crystals, Wiley-VCH Verlag GmbH \& Co. KGaA, Weinheim, Germany, 2014, pp. 1-45.

3 Y. A. Nastishin, H. Liu, T. Schneider, V. Nazarenko, R. Vasyuta, S. V. Shiyanovskii and O. D. Lavrentovich, Phys. Rev. E, 2005, 72, 041711.

4 D. M. Agra-Kooijman, G. Singh, A. Lorenz, P. J. Collings, H. S. Kitzerow and S. Kumar, Phys. Rev. E - Stat. Nonlinear, Soft Matter Phys., 2014, 89, 062504. D. Goldfarb, Z. Luz, N. Spielberg and H. Zimmermann, Mol. Cryst. Liq. Cryst., 1985, 126, 225-246. O. M. Matus Rivas and A. D. Rey, Carbon N. Y., 2016, 110, 189-199. S. Zhou, Liq. Cryst. Today, 2018, 27, 91-108. I. Dierking and A. M. F. Neto, Crystals, 2020, 10, 1-24. A. C. S. Ibanez, E. Marji and Y. Y. Luk, RSC Adv., 2018, 8, 29598-29606.

10 J.-M. Péchiné, A. Meddour and J. Courtieu, Chem. Commun. (Camb)., 2002, 33, 1734-1735.

11 E. Troche-Pesqueira, M. M. Cid and A. Navarro-Vázquez, Org. Biomol. Chem., 2014, 12, 1957-1965. E. Troche-Pesqueira, C. Anklin, R. R. Gil and A. NavarroVázquez, Angew. Chemie - Int. Ed., 2017, 56, 3660-3664. C. M. de Melo Sousa, R. B. Giordani, W. A. M. de Almeida, C. Griesinger, R. R. Gil, A. Navarro-Vázquez and F. Hallwass, Magn. Reson. Chem., 2019, 1-8.
2018, 56, 1176-1182.

A. F. Kostko, B. H. Cipriano, O. a Pinchuk, L. Ziserman, M. a Anisimov, D. Danino and S. R. Raghavan, J. Phys. Chem. B, 2005, 109, 19126-19133.

S. Pieraccini, S. Masiero, A. Ferrarini and G. P. Spada, Chem. Soc. Rev., 2011, 40, 258-271.

O. M. Matus Rivas and A. D. Rey, J. Phys. Chem. B, 2019, 123, 8995-9010.

H. Lee and M. M. Labes, Mol. Cryst. Liq. Cryst., 1984, 108, 125-132.

A. Ferrarini, S. Pieraccini, S. Masiero and G. P. Spada, Beilstein J. Org. Chem., , DOI:10.3762/bjoc.5.50.

L. Bergquist and T. Hegmann, ChemNanoMat, 2017, 3, 863-868.

T. Blümel and H. Stegemeyer, Liq. Cryst., 1988, 3, 195-201. V. A. Burmistrov, I. V. Novikov, V. V. Aleksandriiskii, M. K. Islyaikin, A. S. Kuznetsova and O. I. Koifman, J. Mol. Liq., 2019, 287, 110961.

T. Shirai, M. Shuai, K. Nakamura, A. Yamaguchi, Y. Naka, T. Sasaki, N. A. Clark and K. V. Le, Soft Matter, 2018, 14, 1511-1516.

T. Ogolla, S. B. Nashed and P. J. Collings, Liq. Cryst., 2017, 44, 1968-1978.

J. K. Ando and P. J. Collings, Soft Matter, 2021, 17, 14091414.

L. Tortora and O. D. Lavrentovich, Proc. Natl. Acad. Sci. U. S. A., 2011, 108, 5163-5168.

J. Jeong, Z. S. Davidson, P. J. Collings, T. C. Lubensky and A. G. Yodh, Proc. Natl. Acad. Sci. U. S. A., 2014, 111, 17421747.

C. Peng and O. D. Lavrentovich, Soft Matter, 2015, 11, 10. F. Berride, E. Troche-Pesqueira, G. Feio, E. J. Cabrita, T. Sierra, A. Navarro-Vázquez and M. M. Cid, Soft Matter, 2017, 13, 6810-6815.

C. Pellegrino, M. P. De Santo, L. Spina and F. Ciuchi, $A d v$. Funct. Mater., 2021, 31, 2010394.

C. Yang and Y. Inoue, in Comprehensive Chiroptica Spectroscopy: Applications in Stereochemical Analysis of Synthetic Compounds, Natural Products, and Biomolecules, John Wiley \& Sons, Inc., Hoboken, NJ, USA, 2012, pp. 317353.

H. Yu, C. Welch, W. Qu, C. J. Schubert, F. Liu, G. Siligardi and G. H. Mehl, Mater. Horizons, 2020, 7, 3021-3027.

A. Sugimura and G. R. Luckhurst, Prog. Nucl. Magn. Reson. Spectrosc., 2016, 94-95, 37-74.

C. A. Schneider, W. S. Rasband and K. W. Eliceiri, Nat. Methods, 2012, 9, 671-675.

G. Albano, M. Lissia, G. Pescitelli, L. A. Aronica and L. Di Bari, Mater. Chem. Front., 2017, 1, 2047-2056.

F. D. Saeva and G. R. Olin, J. Am. Chem. Soc., 1973, 95, 7882-7884.

I. Dierking, Textures of Liquid Crystals, Wiley-VCH, 2004.

C. K. McGinn, L. I. Laderman, N. Zimmermann, H. S. Kitzerow and P. J. Collings, Phys. Rev. E - Stat. Nonlinear, Soft Matter Phys., 2013, 88, 062513.

L. J. Prins, P. Timmerman and D. N. Reinhoudt, J. Am. Chem. Soc., 2001, 123, 10153-10163. 